\title{
Location Issues of Thin Shell Parts in the Reconfigurable Fixture for Trimming Operation
}

\author{
Hu Fuwen ${ }^{1}$
}

\begin{abstract}
The location of thin shell parts is always a knotty problem during machining, welding, forming, assembling and inspection operations. This paper mainly focuses on the special location issues in the digital and flexible trimming process for aircraft skins fastened by reconfigurable fixture. Firstly, in view of the dynamic change of effective locators, the "X-2-1" location principle was proposed with reference to the "3-2-1" and "N-2-1" location schemes. Secondly, the standard procedure for solving location parameters was summarized in consideration of location admissibility, holding posture, locator layout and so on. Thirdly, a locating experiment was conducted to investigate the positional accuracy of the reconfigurable fixture and the calculation accuracy of location parameters solution. Fourthly, a quantitative evaluation method to evaluate the dynamic stiffness of the fixturing system was put forward. Moreover, the effects of location layout on the dynamic stiffness were analyzed using the finite element simulation system for the trimming process. A noticeable appearance had been observed that the cliff effect of the dynamic stiffness of the flexible fixturing system may be induced due to the dynamic change of effective locators. Finally, some conclusions and discussion on future works were given.
\end{abstract}

KEYWORDS: Thin shell part, Reconfigurable fixture, Location principle, Dynamic stiffness, Trimming.

\section{INTRODUCTION}

The location of a component is one of the most important aspects to achieve the required accuracy during a machining process. Generally, the proper design of clamping and locating arrangements is a difficult task, considering its workpiece geometry, tool paths, set-up time, production scale, resistance to cutting forces, costs, adaptability and so forth. For a long time, towards fixture design becoming a science rather than an art, and towards achieving automation and intelligence of fixture design activities, many theories, tools and approaches have been introduced by many researchers (Wang et al., 2010; Cecil, 2001; Boyle et al., 2011).

However, most part of this research has concentrated on the fixture design for workpieces which can be regarded as rigid bodies. Actually there are also some low rigidity or flexible components in industrial fields, e.g. aircraft skin panels, automotive cover panels, rocket shells and other thin-wall structures. They cannot be seen as rigid bodies due to their inherent flexibility and inevitable deformation caused by external forces or their own weights. Thereby, their location issues have always been a particularly knotty problem. To overcome it, the single purpose and dedicated fixtures are often employed in practical production. Although this approach would be economical for large scale mass manufacturing (e.g. automobile manufacturing), it would lead to long lead time, a large quantity of tooling, and high production costs under small batch manufacturing environments such as the fabrication of aircrafts and rockets. Therefore, the flexible tooling solutions like reconfigurable fixtures or modular fixtures have been increasingly adopted. These flexible tooling can be changed, reconfigured or reused to suit dimension and shape changes of 
compliant parts. In the past decade or so, a great deal of attention has been increasingly directed toward the design, analysis and synthesis of flexible fixturing systems. There are a number of strategies in order to achieve flexibility (Shirinzadeh, 2002), but this paper mainly focused on the reconfigurable fixtures designed for machining operations of thin shell parts.

According to available documents, the earliest idea of a reconfigurable fixture for thin shell parts trimming process may date back to 1987 (Douglas and Ozer, 1987). About ten years later, the reconfigurable fixture for sheet metal machining was reduced to practice (Proctor, 1998). Afterwards, several commercially available reconfigurable tooling systems were designed and built, for example, the Pogo ${ }^{\circledR}$ flexible tooling system by CNA manufacturing systems (U.S.A.), and the TORRESTOOL ${ }^{\circledR}$ universal holding fixture by the M. Torres group (Spain). Application results from Northrop Grumman indicated that the Pogo ${ }^{\circledR}$ flexible tooling system can cut down setup time by about two-thirds on trimming and hole drilling operations on more than 100 different fuselage skin parts (Koelsch, 1998). But these commercially available reconfigurable tooling systems for holding compliant parts are extremely expensive (Walczyk and Longtin, 1999). Therefore, many researchers and manufacturers are inclined to develop the reconfigurable fixturing devices independently. A reconfigurable fixturing system was specifically designed for the drilling of sheet metal parts, which included a T-slot base plate, vertical supports and locating pins (Youcef-Toumi et al., 1987). A novel reconfigurable modular system was presented for the fixturing of thin-walled flexible objects, which consists of a base plate, and height-adjustable locators and clamps (Sela et al., 1997). A computer-controlled reconfigurable fixturing device for compliant parts, based on a matrix of individually-stoppable pins lowered by a single rigid platen, has been developed (Walczyk and Longtin, 2000). Zhou et al. (2008) designed a flexible tooling system for large-scale aircraft skins by means of the manipulator's drivability. For aircraft skins trimming based on the combination of the reconfigurable fixture and 5-axis gantry type milling machine tool, the author and co-workers have been studying the process planning method, the trimming process simulation, as well as the equipment development $(\mathrm{Hu}$ and Li, 2011; Hu et al., 2012a; Hu et al., 2012b). Besides, to evaluate the milling forces in the trimming process of aircraft skins, the author presented an integrated approach based on the combination of the mechanistic model of milling forces, $3 \mathrm{D}$ finite elements simulation of milling operations, and the support vector regression methodology ( $\mathrm{Hu}$ and $\mathrm{Li}, 2012$ ). Along with the promotion of research, a better systematic understanding of the issue of thin shell components locating in the flexible fixture has been recognized. These would be described as follows.

In the next section, the author would introduce the flexible manufacturing technology for aircraft skins based on the reconfigurable discrete dies and the reconfigurable fixture. And the third section mainly focuses on the distinctive location principle that appears in the trimming process of aircraft skins supported by the reconfigurable fixture. Then the fourth section illustrates the location parameters solution method, considering location allowance, holding posture, locator layout, etc. Then a location experiment was presented to investigate the location accuracy.Subsequently a quantitative evaluation method for the dynamic stiffness of the fixturing system was put forward. Finally, some conclusions and discussion on future works are given.

\section{FLEXIBLE MANUFACTURING MODEL OF AIRCRAFT SKINS}

Aircraft skin panels with the aerodynamic profile are the key components to fabricate fuselage, wings, empennage, and so forth. Generally, skin panels are made of aluminum alloys, however, in the design of new aircrafts, composite panels increasingly tend to be adopted. The accuracy of their shape and dimension and the integrity of their surfaces and edges are extremely stringent. For instance, laser cutting of high-strength aluminum alloys for aerospace applications has been limited, due to its inevitable heat-affected zone (Riveiro et al., 2008). Therefore, the periphery cutting of aircraft skins mainly employs the machining method or the water jet cutting method. In addition to that, skin panels always have low structural rigidity, and complex internal structure and outer shape. These factors make their forming and machining process very difficult. Thereby, the fabrication technology of aircraft skin panels is always a key technology of aircraft manufacturing.

\section{TRADITIONAL FABRICATION PROCEDURE OF AIRCRAFT SKINS}

Traditionally, a typical fabrication process of metal skins is illustrated in Fig. 1. The entire process for any skin part needs 


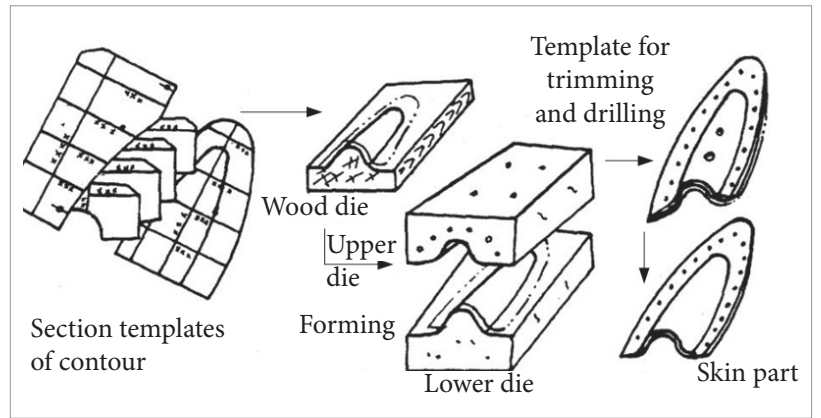

Figure 1. A traditional fabrication procedure of aircraft skins.

a set of dies, templates and other tooling. The setup time of tooling fabrication accounts for approximately 60 to 80 per cent of the cycle time of one part. Furthermore, due to the analog data transfer through the whole process, the accumulative error is often relatively large. Additionally, the whole process has to be executed sequentially rather than concurrently. Once a fault (e.g. springback) occurs on any tooling, repair and rework would be necessary.

\section{FLEXIBLE MANUFACTURING MODEL OF AIRCRAFT SKINS}

In order to improve the fabrication process of metal skins, a flexible, digital and tooling-less manufacturing model was presented, namely, to replace the dedicated dies by discrete multi-point dies and to replace the dedicated trimming template by the reconfigurable fixture (Papazian et al., 2002; Li et al., 2009). As illustrated in Fig. 2, the entire process was accomplished jointly with the digital design, digital tooling, numerical control equipments (e.g. stretch forming machine and CNC machine tool) and digital measure technology. Obviously, the good flexibility of this new manufacturing model can adapt to the dimension and shape changes of aircraft skins, and radically change the traditional fabrication model. As proved in an application experiment performed by Papazian et al. (2002), in this new "tool-less" sheet metal fabrication environment, tooling fabrication time would be reduced by a factor of 8 and labor hours would be reduced to $1 / 3$ of the traditional values.

In the aspect of the flexible stretch forming process, based on the discrete-die concept, undoubtedly, it was David Hardt at MIT and his research group that had done the pioneering work, including the prototype reconfigurable multi-points tooling development and the closed-loop shape control technology (Hardt et al., 1981; Walczyk and Hardt, 1998; Munro and
Walczyk, 2007). On the basis of the original MIT work, since 1996 to 2002, a reconfigurable tooling for flexible fabrication project was sponsored by the Defense Advanced Research Projects Agency. And the project participators included MIT, Northrop Grumman Corporation, Cyril Bath Company, etc. As a result of this project, a commercially available reconfigurable multi-point tooling was designed and built by the Cyril Bath Company in 2002. It was the first production reconfigurable tooling, which had a working volume of $1.06 \times 1.83 \times 0.30 \mathrm{~m}^{3}$ and consists of 2,688 movable pins (Munro and Walczyk, 2007). In China , approximately since 2003, the scholarly research upon the reconfigurable tooling for the stretch forming of aluminum sheet began, and it has been performed chiefly by the Li Mingzhe's research group of Jilin University (Cui et al., 2008; Zhou et al., 2005; Cai et al., 2009) and Li Dongsheng's research team of Beihang University (Chen, 2006; Huang et al., 2008; Yu et al., 2011). At 2007, a larger-size $\left(1.216 \times 1.824 \times 0.305 \mathrm{~m}^{3}\right)$ production reconfigurable tooling was designed and built by the Beijing Aeronautical Manufacturing Technology Research Institute (Zhou, 2007). In short, a persistent and concerted effort by many industry and academic researchers has brought the concept of reconfigurable "discrete-die" tooling to the production environment.

The natures of both the reconfigurable multi-point tooling and the reconfigurable fixture are to use discrete points to approximate a surface. However, as another aspect of the flexible manufacturing model of aircraft skins, the flexible trimming process, based on the reconfigurable fixture, seems to have a reverse development history, namely, from reduction-topractice to academic research. Perhaps there is no such difficult problem as the shape control that remedies the forming defects (e.g. dimpling) which occur in the flexible stretch forming process. Nevertheless, as the chief issue in the development and application of the expensive flexible fixture, the location issue has not been quite clear in academic research. The following factors suggest the particularity and complexity of the workpiece location issues in the flexible trimming process.

- The end effectors are both locators and positioners. Namely, they not only clamp the thin shell part through the vacuum absorption, but also locate the part consistently to prevent the possible deformation. Moreover, the locating and clamping are synchronous.

- When the "flexibility" of multi-point supporting would encounter the "elasticity" of the low-rigidity thin shell part, the location stability would be difficult to assure. Additionally, 


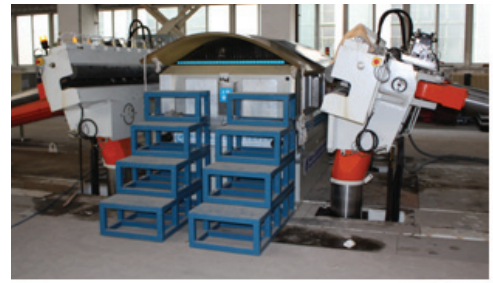

Stretch forming over the reconfigurable tooling

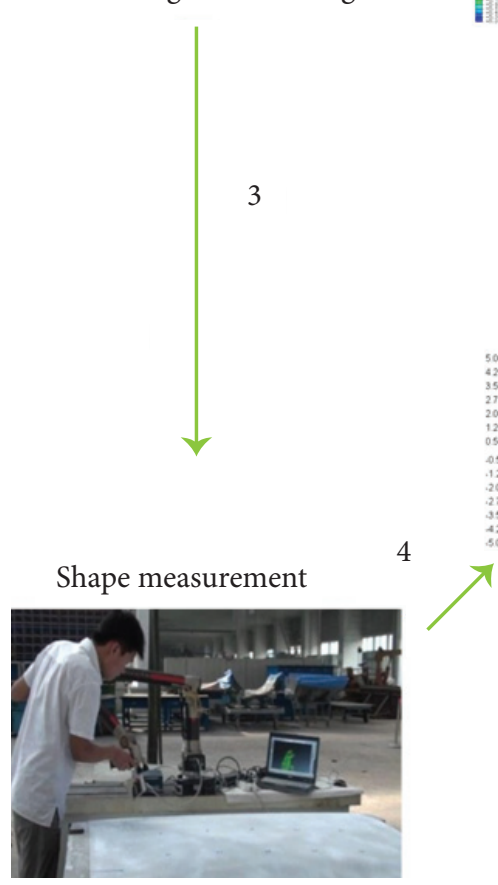

\section{2}

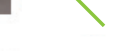

10

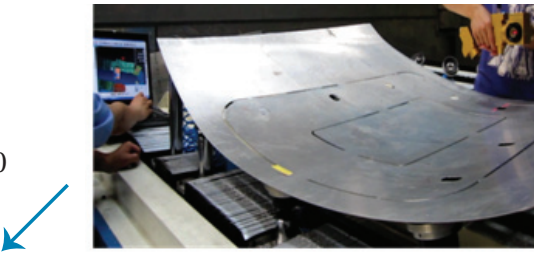

Shape measurement

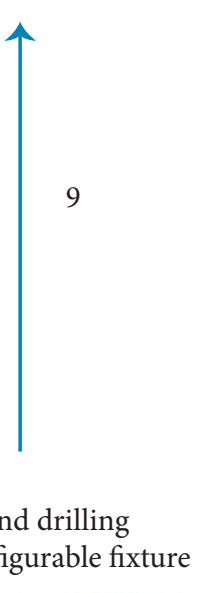

6
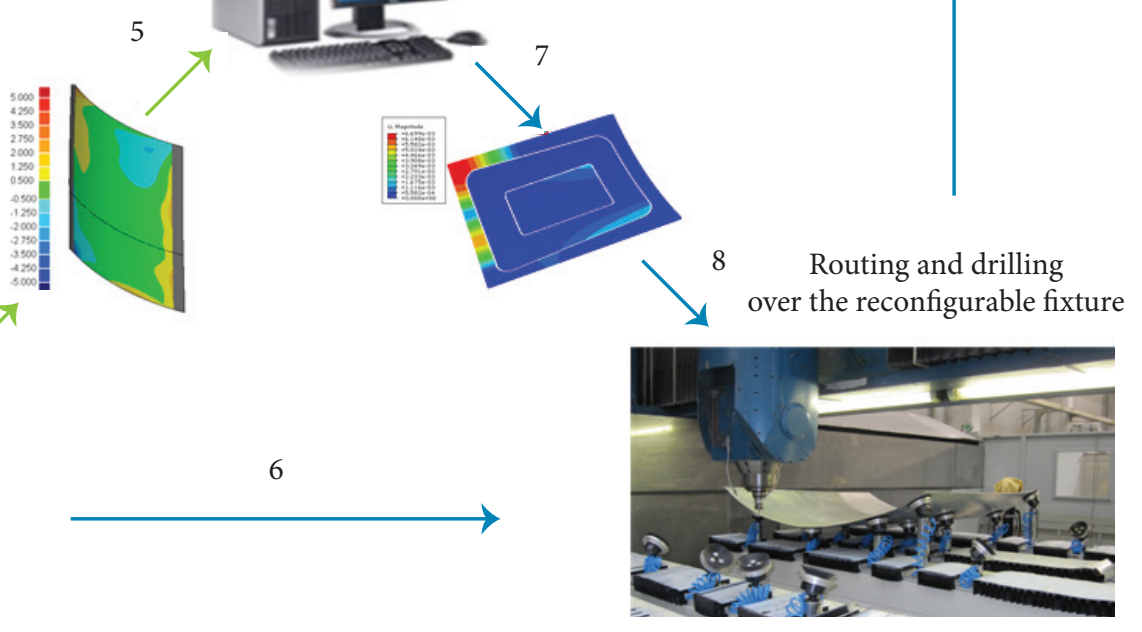

Main contents of step [1] include pin height calculation, stretching trajectory calculation and springback compensation; In step[2] the sheet metal would be formed over the reconfigurable multi-point tooling: Then, the geometrical precision would be measured via laser scanning; In step[4] the error distribution can be obtained after data processing; If the formation precision cannot meet the requirements, step [1]-[4] begin the cycle again; Otherwise, the surface part would be located in the

reconfigurable fixture for machining operations; Certainly, before loading the surface part, the location parameter of the reconfigurable fixture would be solved; In step [8] the surface part would be machined over the reconfigurable multi-point tooling; Then, the machining precision would be measured via laser scanning; In step [10] the error distribution can be obtained after data processing; If the machining precision cannot meet the requirements, step [7]-[10] begin the cycle again.

Figure 2. Flexible manufacturing model of aircraft skins.

with the gradual separation of the thin shell part, and with the loading of the periodical milling forces, the process stability would also be difficult to assure. The whole process stability turns out to be a particularly knotty problem.

- The main task of location layout is to determine the positions and numbers of vacuum end effectors. Two types of restriction should be taken into account: one is geometric constraint, which ensures there is no interference between any two adjacent actuator rods, between any two adjacent lateral frames, and between the machining cutter and the vacuum end effectors; another one is physical constraint, which mainly considers the clamping and processing stability. The method for planning feasible location layout should be studied.

\section{LOCATION PRINCIPLES}

\section{ADEQUATE LOCATION}

As it is known, a rigid body has six movement degrees of freedom ( 3 linear and 3 rotary ones) in a three-dimensional space. If a workpiece can be regarded as a rigid body, it is necessary to arrest the DOF (degrees of freedom) $(\leq 6)$, which 
affects the machining accuracy. However, due to low rigidity and easy deformation, the thin shell part cannot be considered as a rigid body. As illustrated in Fig. 3, if the elastic surface part is supposed to consiste of $\mathrm{n}$ mass points, it would be essential to limit all DOF of $\mathrm{n}$ mass points to avoid machining errors. Theoretically, a mass point has three DOF (3 linear), and $\mathrm{n}$ tend to be the infinity of integers. Therefore, the only way to limit all 3n DOF entirely is "surface location" provided by the dedicated hard surface fixture (Fig. 4). Moreover, for the cutter penetration, trimming paths should be slotted on the surface of the rigid fixture. Also the trimming or drilling operations can be done by manual cutters, if the plate thickness is less than $2 \mathrm{~mm}$, otherwise by the numerical control machine tool.

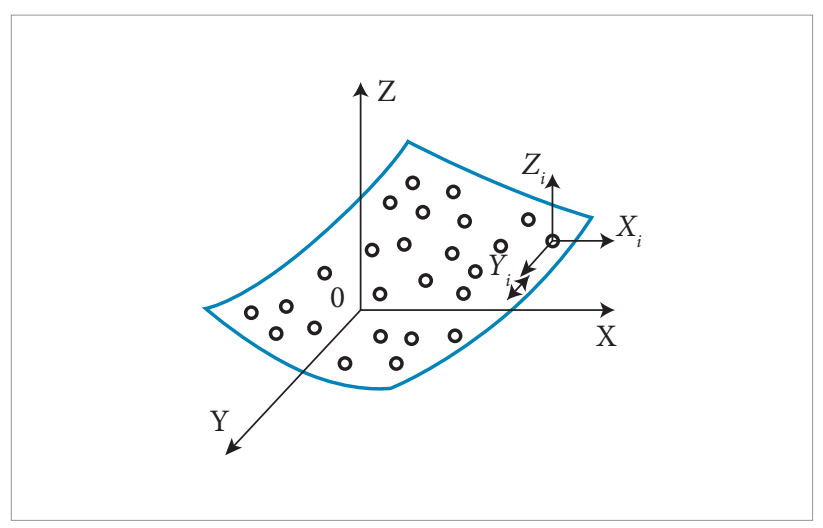

Figure 3. Discrete points model of a thin shell part.

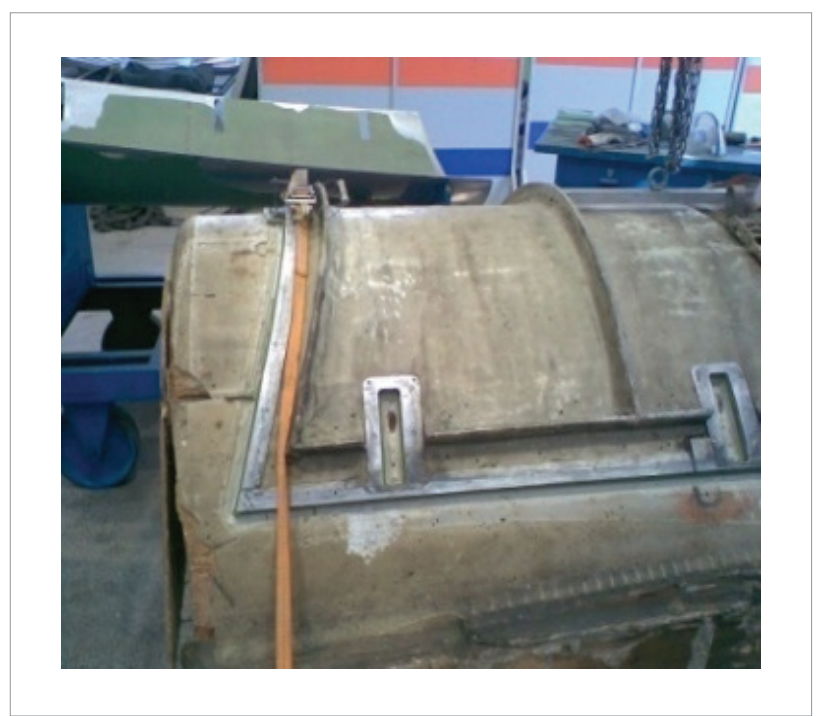

Figure 4. Manual trimming of aircraft skins on the dedicated tooling.
Apparently, when using the reconfigurable fixture to locate the thin shell part (Fig. 2), only the DOF of the mass points close to the vacuum end effectors can be partly restricted. Due to the loaded milling forces and inherent low rigidity, especially in the normal direction, vibration would be unavoidable. In this case, if the required machining accuracy would not be affected, the location status also can be called adequate location. Evidently, adequate location is one of the essential requirements for guaranteeing the machining accuracy.

\section{LOCATION SCHEMES}

Generally, for a rigid part, the basic location concept is to arrest the motion that may affect the machining accuracy. Specifically, for a prismatic part without an existing hole, the "3-2-1" location principle can arise proper arresting of all the motions. As illustrated in Fig. 5, "3" refers to 3 locators (passive fixture elements) on the primary datum surface; and " 2 " refers to 2 locators on the secondary datum surface; then " 1 " refers to 1 locator on the tertiary datum surface. However, the 3-2-1 principles can only be applied for prismatic-workpiece fixturing, and the three perpendicular datum planes and their corresponding features must be well defined. For a prismatic part with an existing hole, the most efficient way of locating the workpiece is to apply three supports and a single internal locator to restrict nine movements at once. And for nonprismatic parts, the fixturing techniques are often dependent on the workpiece shape. Moreover, due to the complex nature of workpiece geometry, there are no generalized fixture-design principles for nonprismatic parts.

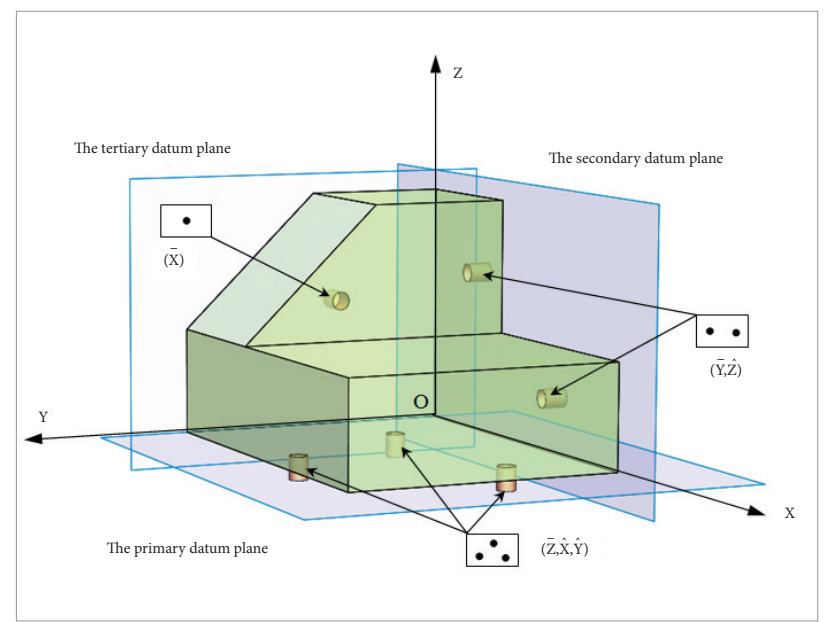

Figure 5. "3-2-1" location principle for a prismatic part without an existing hole. 
The basic "3-2-1" location scheme for rigid parts may not be suitable for thin shell and compliant objects, due to their inherent flexibility and low rigidity. In such case, the "N-2-1" locating scheme (Fig. 6) was formally introduced for deformable sheet metal parts, as compared to the widely accepted "3-2-1" principle for rigid bodies, where $\mathrm{N}$ supports are required in order to support the metal sheet part in the primary datum direction to avoid excessive deflection (Cai et al., 1996). It must be pointed out that the "N-2-1" locating scheme was presented considering that of when drilling forces or resistance spot welding forces were induced. Therefore, the "N-2-1" location principle is appropriate when the reconfigurable fixture are used for inspection, laser scribing and for the drilling process.

Nevertheless, in case of trimming process, the "N-2-1" locating scheme can not reveal the appearance that some locators gradually lose their effectiveness. Both the researches of Walczyk and Longtin (1999) and the initial research of the author ( $\mathrm{Hu}$ and $\mathrm{Li}, 2011$ ) neglected this singular detail. As illustrated in Fig. 7, at the beginning of trimming, there are X locators which take action; while with the separation of the desired shape from the blank, there are Xs locators that gradually lose their effectiveness. Until the end, only Xw locators continue to work. In short, the locators are always changing due to the workpiece separation. The author called this particular location scheme "X-2-1" location principle. "X" refers to the locators of dynamic changes. In order to guarantee the stability of the whole trimming process, designing the locators' initial layout should take the dynamic change into account. Otherwise, the trimming operation may failure due to machining errors or other accidents.

\section{LOCATION PARAMETERS SOLUTION}

Location parameters refer to the desired positions that vacuum end effectors and reference positioners should reach,

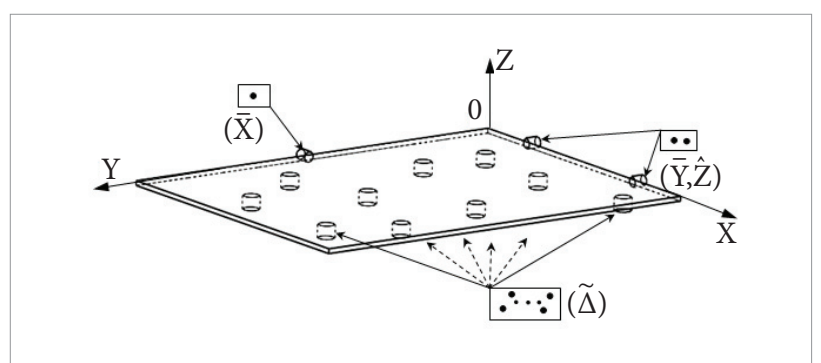

Figure 6. "N-2-1" location principle for deformable metal sheet parts in drilling and welding. according to the dimension and shape of the surface part. The nature of location parameters solution is to transfer the aircraft skins digital model into the numerical control data, which drive the reconfigurable fixture into quick and accurate reconstruction. Two different solution methods have been presented by the author: one is based on the geometric structures of locators and positioners (Hu et al., 2012a); another is based on the virtual locating and positioning (Hu et al., 2012b). In this paper, the standard procedure of location parameters solution is summarized as shown in Fig. 8. In a previous research by the authors (Hu et al., 2012a; Hu et al., 2012b), the procedures of holding posture design, locators layout design and location parameters solution were discussed in details. Whereas the location admissibility was omitted in previous researches, it would be described emphatically as follows.

Location admissibility indicates whether the thin shell part can be located and supported properly according to the configuration of the reconfigurable fixture and the part model. Specifically, the listed aspects should be taken into account:

- The dimensions of the thin shell part are beyond the working stroke of the reconfigurable fixture?

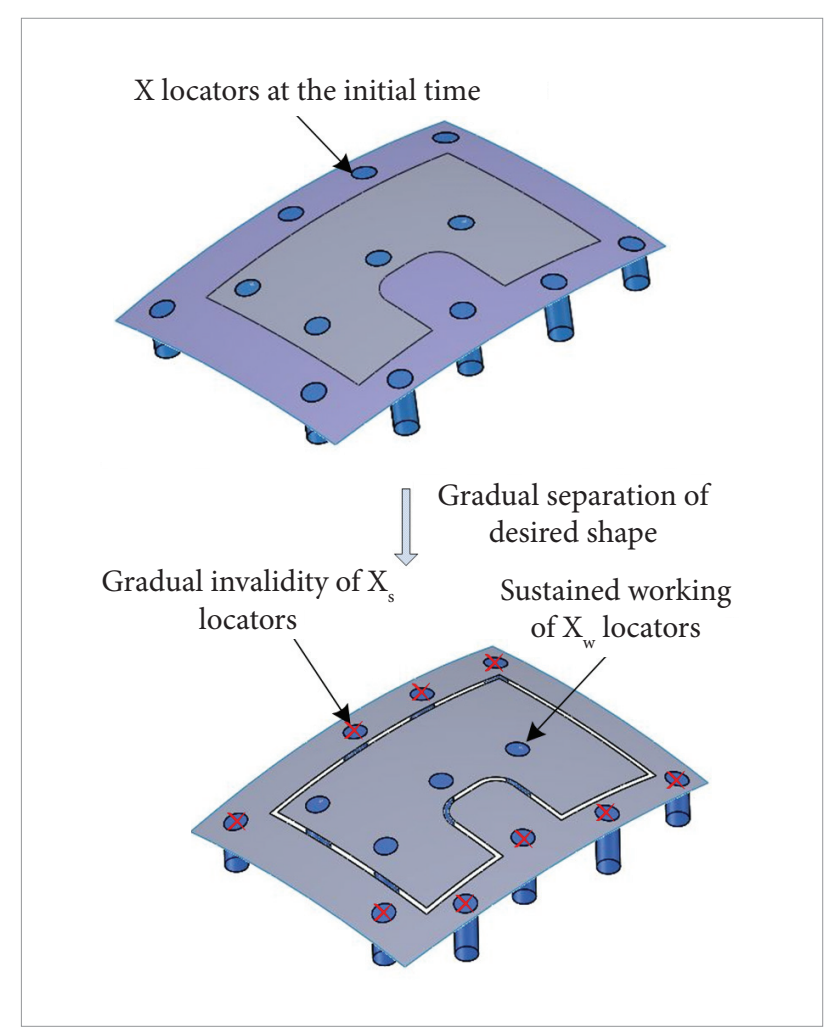

Figure 7. "X-2-1" location principle for thin shell parts during flexible trimming process. 
- Due to the part dimensions and the cutting trajectory, the locators that can be arranged are too few?

- The local curvature of the thin shell part is beyond the maximum allowable swing angle of end effectors?

The first two aspects are comparatively easy to judge and address, according to the part model and the features of the reconfigurable fixture. As for the third point, when the part surface is adsorbed onto the vacuum suction of end effectors, the centre line of the end effector should be aligned to the normal direction $n_{i}$ of the contact center point $P_{i}$ on the locating surface. Evidently, the center line would always be in the maximum conical angle $\theta_{\text {max }}$, when the end effector swings freely to suit the local curvature of the thin shell part. After the holding posture is defined, as illustrated in Fig. 9, transfer the normal vector $n_{i}$ of $P_{i}$ point in the workpiece coordinate system into the normal vector $n_{i}^{L}$ in the end effector coordinate system. Then figure out the angle $\theta$ between $n_{i}^{L}$ and the axis $Z_{L}$ If

$\theta \leq \theta_{\max }$

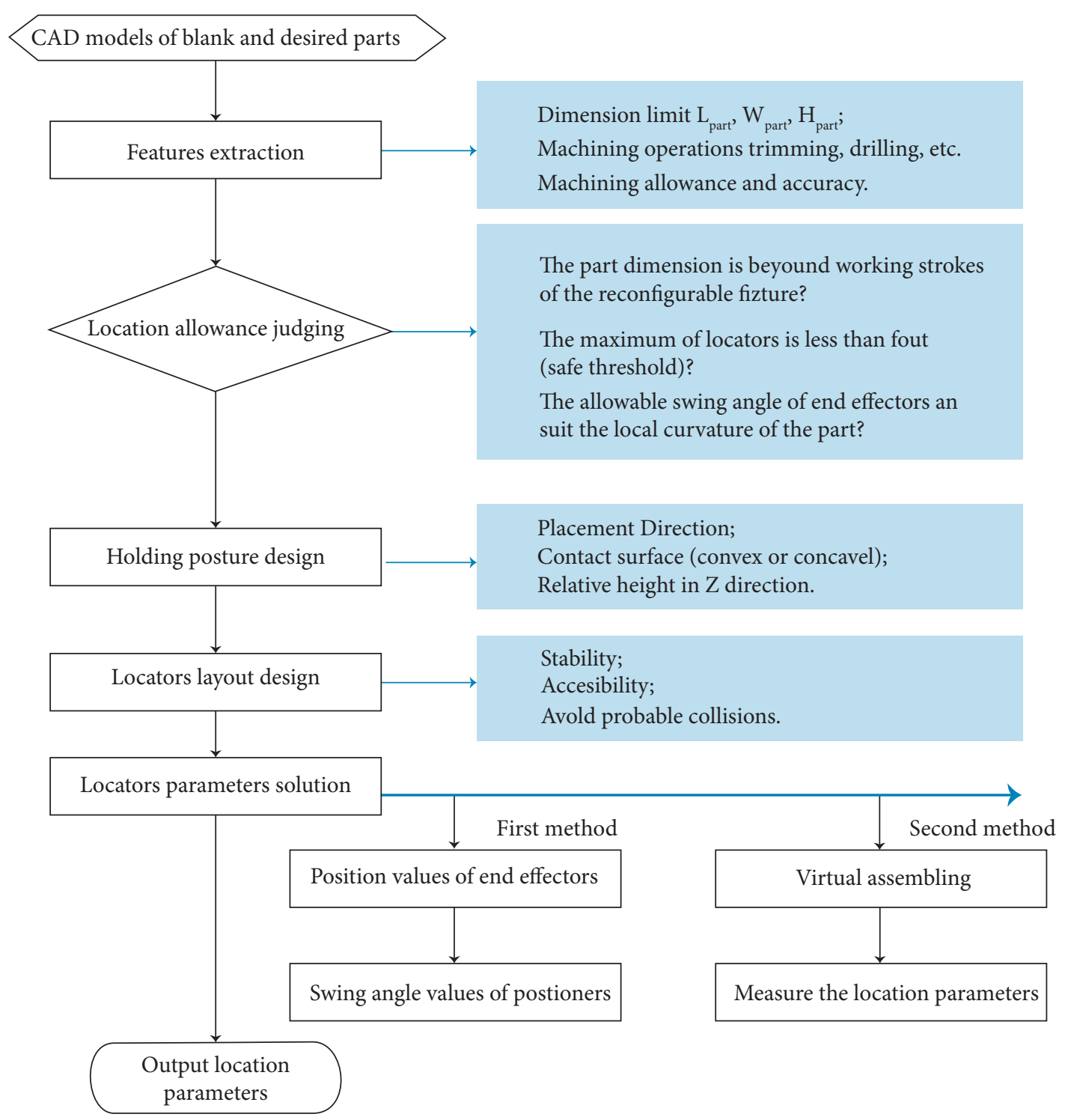

Figure 8. Location parameters solution procedure. 
the end effectors can be located at this point. Otherwise, the end effectors cannot suit the local curvature of this point. For instance, the curvature of the leading-edge skin components is often great; thereby the free swing holding cup cannot be available. Therefore, the commercially available reconfigurable tooling system would generally provide a quick-change style adapter such as extension arms (Fig. 10).

For the automation of location parameters solution, a special location parameters solution software system (Flextrimming

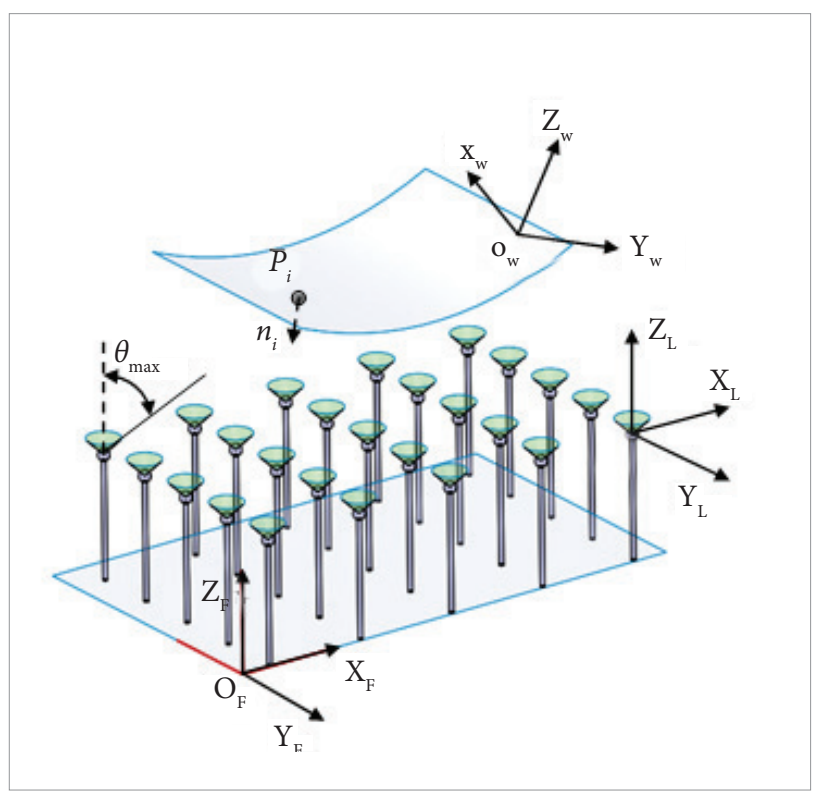

Figure 9. Local curvature and the maximum conical angle.

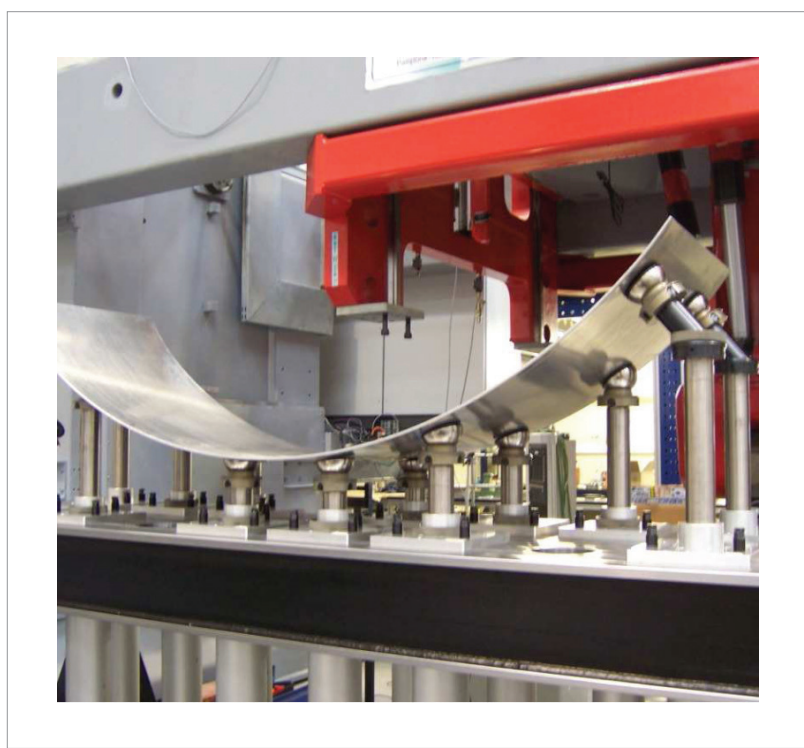

Figure 10. Quick-change style adapter. system) has been improved and seamlessly integrated into the CATIA V5 software via the component application architecture (CAA) development kit, a component object model-like interface. The Flextrimming system can, in sequence, perform the location parameters solution procedure interactively: features extraction, locating allowance judging, holding postures design, locators layout and location parameters solution.

\section{LOCATION ACCURACY}

The geometrical accuracy of a machined feature on a workpiece depends on, partially, the fixture's ability to precisely locate the workpiece, which is in fact related to the locator's configurations and positional accuracy of each locator. The reconfigurable flexible, developed independently by our research group, maintains a positioning accuracy of $0.1 \mathrm{~mm}$ and a repeatability of $0.05 \mathrm{~mm}$. On the other hand, the solution accuracy of location parameters can reach $0.1 \mu \mathrm{m}$. Namely, the final location accuracy depends on both the hardware and the software systems, and in order to validate the final location accuracy, an aluminum alloy skin part with a thickness of $1.27 \mathrm{~mm}$ was selected and located on the reconfigurable flexible fixture (Fig. 11). Then the located workpiece was measured using the API Tracker3 laser tracker (Fig. 12).

Comparative analysis between the digital model and the measurement data were performed, and the obtained error contour is shown in Fig. 13. The final errors may be caused by the multiple effects including measurement error, forming errors and location errors. Thereby errors distribution was in the reasonable scope and it can be acceptable in practical productions. Meanwhile it may be observed that there is little deformation caused by the vacuum absorption of end effectors. Though the aircraft skin is very thin and poorly rigid, its local rigidity is enough to make the end effectors swing freely to automatically adjust to the local curvatures change of the thin shell part.

\section{DYNAMIC STIFFNESS OF THE PROCESS SYSTEM}

\section{EVALUATION OF DYNAMIC STIFFNESS}

Dynamic stiffness is a measure of the machining system's ability to dampen the vibrations from input forces, which would 
directly affect machining accuracy and stability. Theoretically, under the action of machining forces, the whole deformation of the

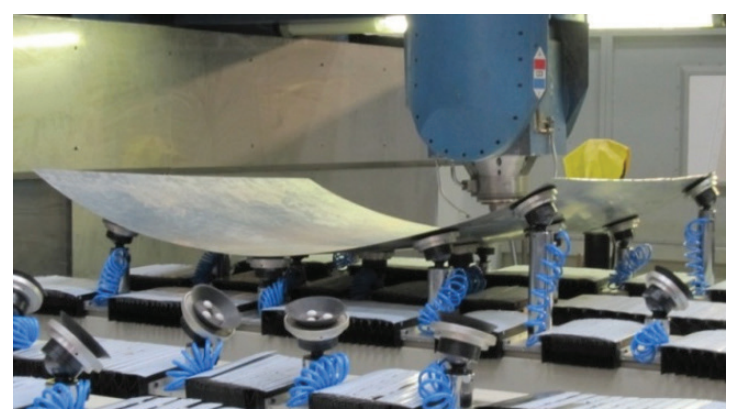

Figure 11. Locating set-up.

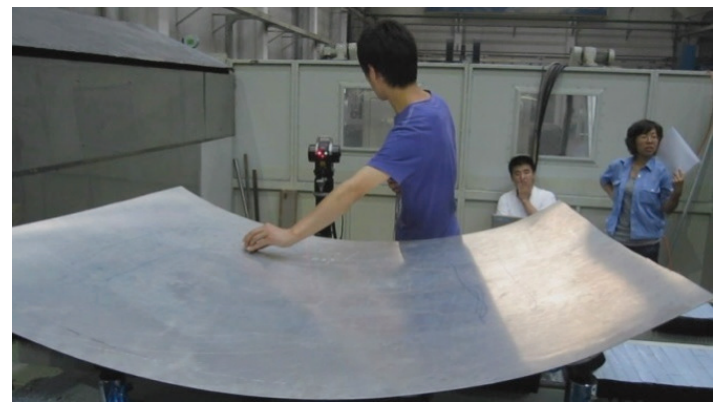

Figure 12. Measurement via laser tracker.

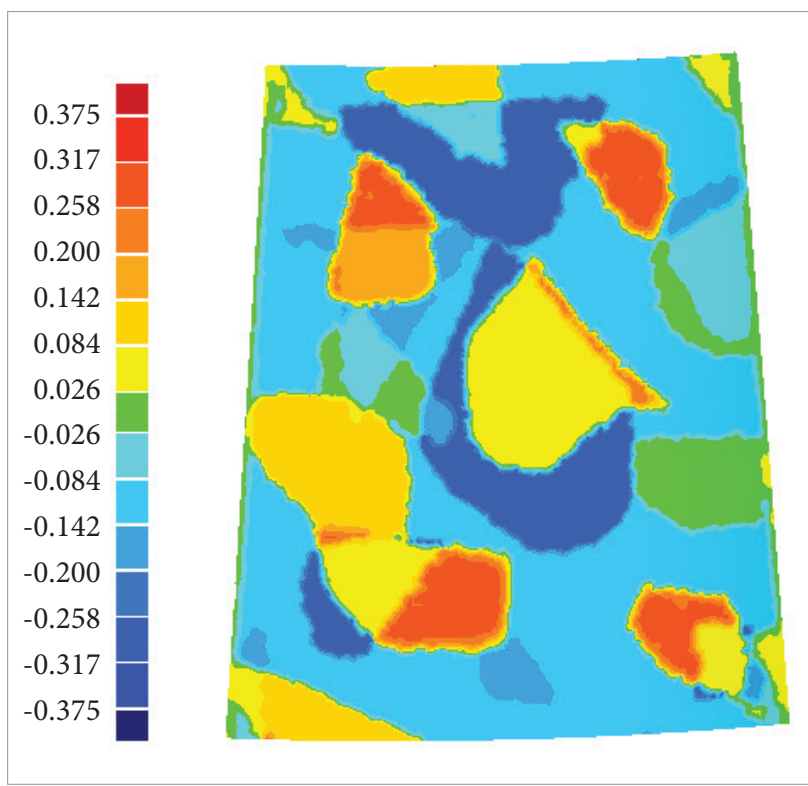

Figure 13. Error distribution contour. process system is caused by each individual component including the deformations of machine tool, cutter, fixture and workpiece. In fact, the thin shell part has relatively poorer stiffness, thus the deformation of thin shell part is the largest part of the whole deformation of the process system. Therefore, other deformations are neglected and only the workpiece deformation is considered.

As previously mentioned, the surface part can be regarded as the constitution of discrete points. Then the points close to end effectors could be fully restricted and the displacements of other points would vibrate along with the trimming process. Obviously, the closer the point is to the vacuum adsorption area, the less its vibration will be. Conversely, the farther the point is to the cutting path, the greater its vibration will be. In order to evaluate the dynamic stiffness of the whole fixturing system, $n$ points near to the cutting paths was selected evenly from nodes on the elements as reference points (Fig. 14) and the dynamic stiffness is defined as follows,

$$
\begin{aligned}
& K_{t}=\frac{F_{t}}{\Delta\left(F_{t}, t\right)}=\frac{1}{1 / k_{1 t}+1 / k_{2 t}+\cdots+1 / k_{n t}} \\
& \delta_{i}\left(F_{t}, t\right)=\frac{F_{t}}{k_{i t}}
\end{aligned}
$$

$$
\Delta\left(F_{t} t\right)=\delta_{1}(F, t)+\delta_{2}(F, t)+\cdots+\delta_{n}(F, t)
$$

Where $F_{t}$ denotes the external loads, and the subscript $t$ is the trimming time; $\delta_{i}\left(F_{p}, t\right)$ is the instantaneous displacement of the $\mathrm{i}$-th reference point; and $\Delta\left(F_{t} t\right)$ is the instantaneous displacement of the whole process system.

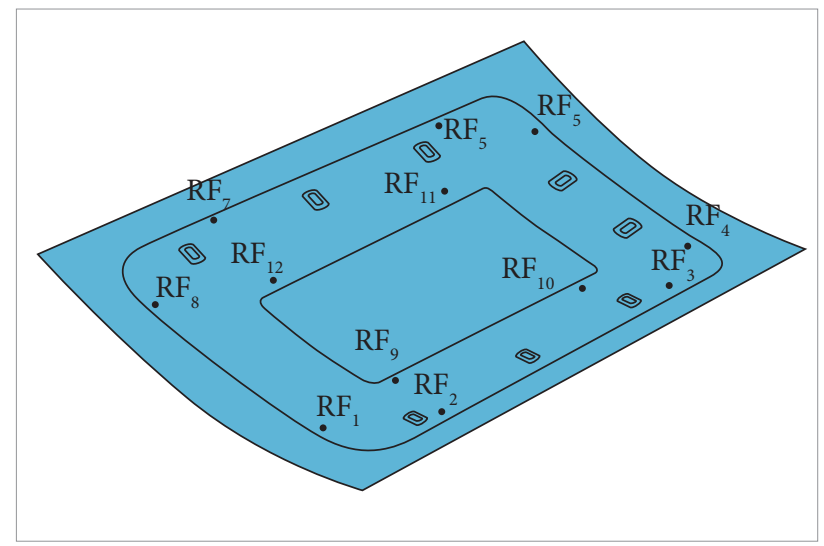

Figure 14. Reference points to evaluate the dynamic stiffness. 


\section{EFFECTS OF LOCATION LAYOUT ON DYNAMIC STIFFNESS}

In order to analyze the effects of location layout on the dynamic stiffness, the finite element simulation was performed using the specially developed system for the flexible trimming process ( $\mathrm{Hu}$ et al., 2012b).The basic principle is to sequentially "kill" the elements on the trimming paths in order to simulate the separation process of the desired part. Meanwhile, the milling forces are loaded on the elements that would be "killed" in the next step. As regards the milling forces magnitude, the proposed method ( $\mathrm{Hu}$ and $\mathrm{Li}, 2012$ ) was employed. Hereby, the displacements of the reference points can be solved dynamically. The whole procedure is illustrated in Fig. 15.

For the part as shown in Fig. 14, to follow the proposed steps (Fig. 8), five different location layouts were designed and the location parameters were listed in Table 1. The reference coordinate system was given in the Fig. 16. Therefore, the relative distance can be distinguished easily by Table 1 and Fig. 16.

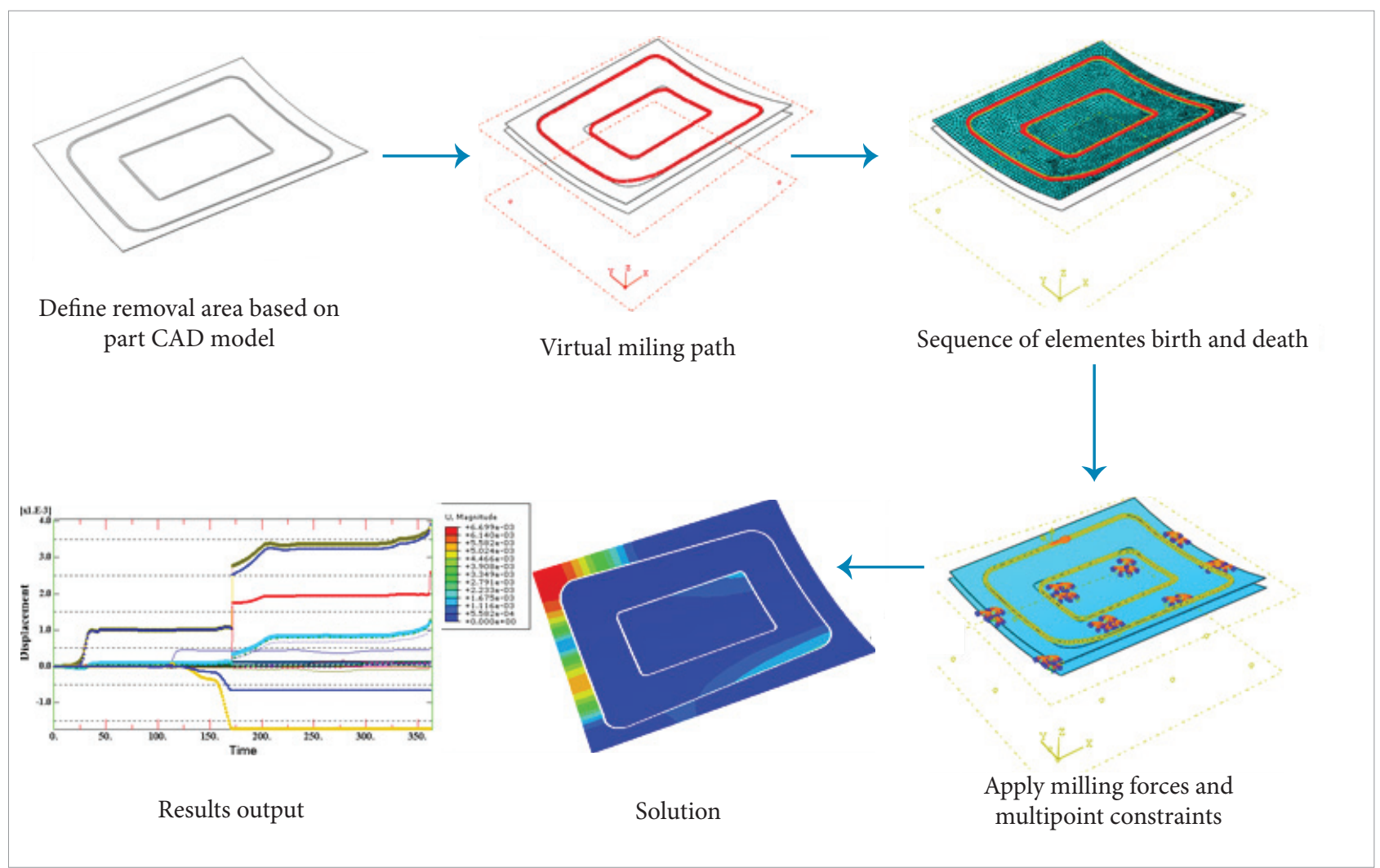

Figure 15. Simulation procedure for trimming process.

Table 1. Location layout $\mathrm{mm}$.

\begin{tabular}{|c|c|c|c|c|c|c|c|c|c|c|c|c|}
\hline Layout No. & $x_{1}$ & $x_{2}$ & $x_{3}$ & $y_{11}$ & $y_{12}$ & $y_{13}$ & $y_{21}$ & $y_{22}$ & $y_{23}$ & $y_{31}$ & $y_{32}$ & $y_{33}$ \\
\hline $1 \#$ & 169 & 579 & 1112 & 39 & 481 & 855 & 175 & 583 & 991 & 39 & 481 & 923 \\
\hline $2 \#$ & 46 & 579 & 1030 & 141 & 617 & 991 & 39 & 447 & 991 & 175 & 549 & 991 \\
\hline $3 \#$ & 46 & 456 & 1153 & 107 & 515 & 923 & 141 & 515 & 957 & 39 & 481 & 923 \\
\hline $4 \#$ & 39 & 481 & 923 & 175 & 549 & 923 & 39 & 481 & 991 & 39 & 447 & 991 \\
\hline $5 \#$ & 128 & 661 & 1112 & 141 & 583 & 991 & 73 & 617 & 991 & 39 & 549 & 957 \\
\hline
\end{tabular}



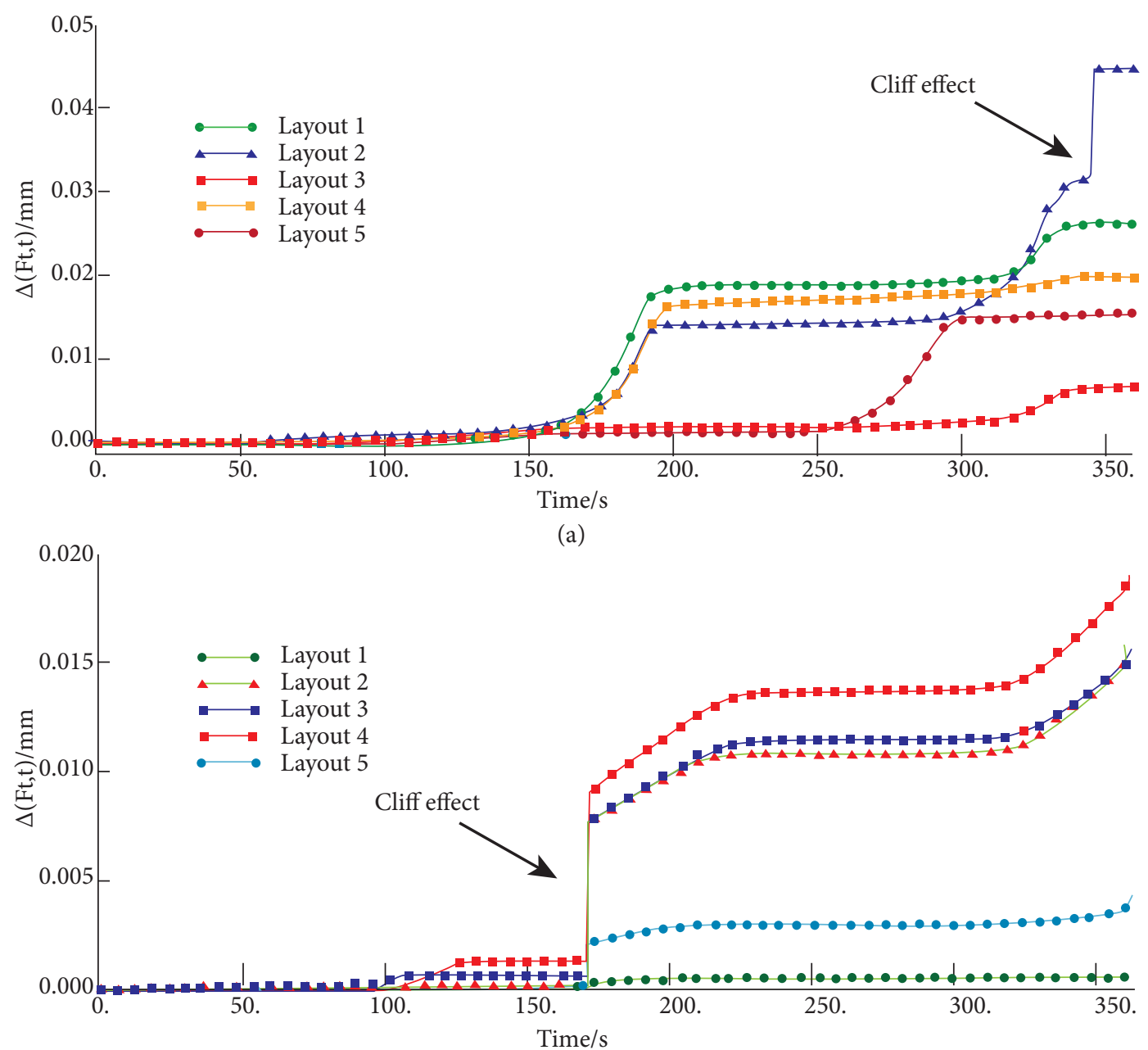

(b)
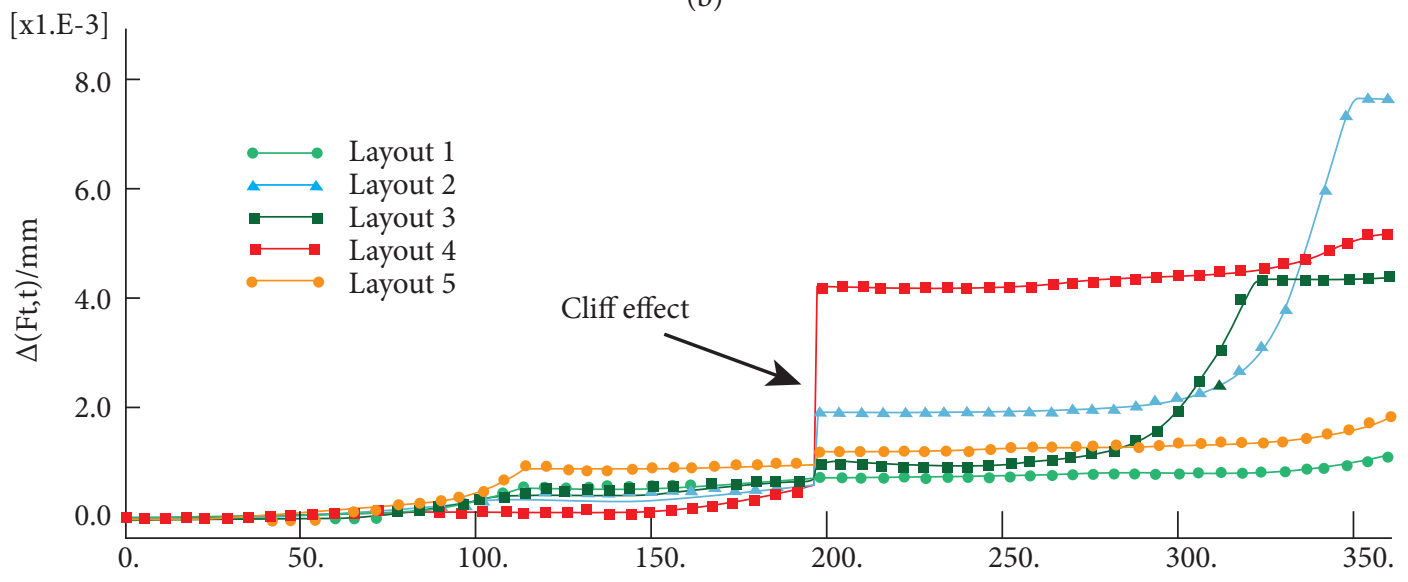

Time/s

(c)

Figure 16. Simulation procedure for trimming process. Cutting parameters: (a) $n=10000 \mathrm{r} / \mathrm{min}, a P=1.27 \mathrm{~mm}$, $f Z=0.1 \mathrm{~mm}$; (b) $n=10000 \mathrm{r} / \mathrm{min}, \mathrm{aP}=2.54 \mathrm{~mm}, \mathrm{fZ}=0.1 \mathrm{~mm}$; (c) $\mathrm{n}=10000 \mathrm{r} / \mathrm{min}, \mathrm{aP}=3.5 \mathrm{~mm}, \mathrm{fZ}=0.1 \mathrm{~mm}$. 
From the finite element situation results (Fig. 16), it was quite easy to tell that the first and fifth layouts were the best arrangements when compared to the other three layouts. The symbols $n, a_{p}$ and $f_{Z}$, respectively, denote the speed of spindle, the part thickness and the feed per tooth. The good location layout could maintain the dynamic stiffness along with the trimming time. Especially, with the decreasing of the part thickness, the location layout has an increasing effect on the dynamic stiffness of the whole process system.

Another noticeable appearance, as marked in the Fig. 16, should not be ignored, i.e., the cliff effect of the instantaneous displacement of the reference nodes. In the case, the dynamic stiffness of the fixturing system would drop suddenly, due to some locator, becoming valid. At this time, dramatic vibrations may occur and the machining error would be induced. With the decrease of the part thickness, the effect of layout on the dynamic stiffness would increase. However, the effect of layout on the time of the cliff effect occurrence may not be obvious. If the location layout was designed properly, the cliff effect could be restrained within narrow limits, such as the first and fifth layouts.

Therefore, when planning the location layout, the cliff effect case should be avoided.

\section{Conclusions}

The numerical control trimming process for aircraft skins in the reconfigurable fixture is a digital and flexible fabrication technology. However, due to the multi-point location, low rigidity of thin shell part and the ongoing separation of desired contours, this promising technology becomes a knotty operation to guarantee good workpiece-holding stability and machining accuracy. This paper mainly contributes to the following issues:

- The unique location schema, namely the "X-2-1" location principle, was revealed in view of the gradual reduction of effective locators of the reconfigurable fixture during trimming process. This work may perfect the location principles of low rigidity parts. Meanwhile, the "discrete points" model of thin shell parts was introduced; thereby it would help to figure out the nature of thin shell part location (i.e., to restrict the degrees of freedom of the discrete mass points) and the quantitative evaluation for the dynamic stiffness of the fixturing system (i.e., to calculate the displatments of the referred nodes).

- The location parameters solution method was further summarized. Especially, the location admissibility was mainly presented; this work improved the location design procedure. In addition, the location accuracy was discussed through a locating experiment in order to validate the location parameters solution accuracy as well as the positional accuracy of the reconfigurable fixture.

- A quantitative evaluation method of the dynamic stiffness of the fixturing system was put forward. This work provided a criterion for the quantitative analysis of location layout. Moreover, the effects of location layout on the dynamic stiffness of the flexible fixturing system were analyzed using the finite element simulation system for the trimming process. Noticeably, the cliff effect of the dynamic stiffness can be observed from the simulation results. This case should be alleviated or avoided through the optimization of location layout.

\section{ACKNOWLEDGEMENTS}

Hu Fuwen would like to thank the Doctor Foundation Project of North China University of Technology and the Science and Technology Innovation Platform Project of Beijing Municipality (PXM2014_014212_000016) for their financial support, and Li Dongsheng and Xu Honghai for their constructive opinions.

\section{REFERENCES}

Boyle, I., Rong, Y. and Brown, D.C., 2011, "A Review and Analysis of Current Computer-Aided Fixture Design Approaches", Robotics and Computer-Integrated Manufacturing, Vol. 27, No. 1, pp. 1-12. doi: 10.1016/j.rcim.2010.05.008.
Cai, W., Hu, S.J. and Yuan, J.X., 1996, "Deformable Sheet Metal Fixturing: Principles, Algorithms, and Simulations", Journal of Mechanical Design, Vol. 118, No. 3, pp. 318-324. doi: 10.1115/1.2831031. 
Cai, Z.Y., Wang, S.H., Xu, X.D. and Li, M.Z., 2009, "Numerical Simulation for the Multi-Point Stretch Forming Process of Sheet Metal", Journal of Materials Processing Technology, Vol. 209, No. 1, pp. 396-407. doi: 10.1016/j.jmatprotec.2008.02.010.

Cecil J., 2001, "Computer-Aided Fixture Design - a Review and Future Trends", The International Journal of Advanced Manufacturing Technology, Vol. 18, No. 11, pp. 790-793. doi: 10.1007/s001700170004.

Chen, L., 2006, "Research and Key Techniques on the Establishment of Reconfigurable Flexible Stretch-Formed Die", Beihang University, Beijing, China.

Cui, X., Xu, X., Li, G., Li, M. and Cai, Z., 2008, "Development and Application of the Multi-Point Stretch-Forming Equipment for Aircraft Skins", China Metal forming Equipment \& Manufacturing Technology, Vol. 43, No. 3, pp. 35-37. doi: 10.3969/j.issn.1672-0121.2008.03.011.

Douglas, W.A. and Ozer, T., 1987, "Universal Holding Fixture", U.S.A patents 4684113

Hardt, D.E., Olson, B.A., Allison, B.T. and Pasch, K., 1981, "Sheet Metal Forming With Discrete Die Surfaces", Ninth North American Manufacturing Research Conference Proceedings, pp. 140-144.

Hu, F. and Li D., 2011, "Process Planning and Simulation Strategies for Perimeter Milling of Thin-Walled Flexible Parts Held by Reconfigurable Fixturing System", IEEE 2011 Third International Conference on Measuring Technology and Mechatronics Automation, Vol. 2, pp. 922926. doi: 10.1109/ICMTMA.2011.513.

Hu, F. and Li D., 2012, "Modelling and Simulation of Milling Forces Using an Arbitrary Lagrangian-Eulerian Finite Element Method and Support Vector Regression", Journal of Optimization Theory and Applications, Vol. 153, No. 2, pp. 461-484. doi: 10.1007/s10957-011-9927-y.

Hu, F., Li, D., Li, X. and Zhu, M., 2012a, "Locating Simulation for Aircraft Skins NC Trimming Based on Flexible Holding Fixture", Computer Integrated Manufacturing Systems, Vol. 18, pp. 993-998.

Hu, F., Li, D., Li, X. and Zhu, M., 2012b, "Process Planning of Aircraft Skins NC Trimming Based on Reconfigurable Fixture", Journal of Beijing University of Aeronautics and Astronautics, Vol. 38, pp. 675-680.

Huang, L., Li, D. and Luo, H., 2008, "Research on Closed-Loop Shape Control System of Stretch Forming Over Reconfigurable Tooling", Journal of Plasticity Engineering, Vol. 15, No. 6, pp. 38-42.

Koelsch, J.R., 1998, "Hold It", Machine Shop Guide, Vol. 3, pp. 26-33.

Li, D., Luo, H., Wang, L. and Li, X., 2009, "Numerical Forming Technology of the Aircraft Skin", Journal of Plasticity Engineering, Vol. 16, pp. 61-65.

Munro, C. and Walczyk, D., 2007, "Reconfigurable Pin-Type Tooling: a Survey of Prior Art and Reduction to Practice", Journal of ManufacturingScienceandEngineering, Vol. 129, No. 3, pp. 551-565. doi: 10.1115/1.2714577.
Papazian, J.M., Anagnostou, E.L., Christ, R.J., Hoitsma, Jr.D., Ogilvie, P. and Schwarz, R.C., 2002, "Tooling for rapid sheet metal parts production", 6th Joint FAA/DoD/NASA Conference on Aging Aircraft, San Francisco, CA, USA

Proctor, P., 1998, "Pogo Fixtures Enhance Tooling Flexibility", Aviation Week \& Space Technology, Vol. 149, No. 8, pp. 50-51.

Riveiro, A., Quintero, F., Lusquiños, F., Pou, J. and Pérez-Amor, M., 2008, "Laser Cutting of 2024-T3 Aeronautic Aluminum Alloy", Journal of Laser Applications, Vol. 20, pp. 225-230. doi: $10.2351 / 1.2995769$.

Sela, M.N., Gaudry, O., Dombre, E. and Benhabib, B., 1997, "A Reconfigurable Modular Fixturing System for Thin-Walled Flexible Objects", The International Journal of Advanced Manufacturing Technology, Vo1. 13, No. 9, pp. 611-617. doi: 10.1007/ BF01350819.

Shirinzadeh, B., 2002, "Flexible Fixturing for Workpiece Positioning and Constraining", Assembly Automation, Vol. 22, No. 2, pp. 112-120. doi: 10.1108/01445150210423143

Walczyk, D.F. and Hardt, D.E., 1998, "Design and Analysis of Reconfigurable Discrete Dies for Sheet Metal Forming", Journal of Manufacturing Systems, Vol. 17, No. 6, pp. 436-454. doi: 10.1016/ S0278-6125(99)80003-X

Walczyk, D.F. and Longtin, R.S., 1999, "Fixturing of Compliant Parts Using a Matrix of Reconfigurable Pins", Journal of Manufacturing Science and Engineering-transactions of The Asme, Vol. 122, No. 4, pp. 766772. doi: 10.1115/1.1314599.

Wang, H., Rong, Y.K., Li, H. and Shaun, P., 2010, "Computer Aided Fixture Design: Recent Research And Trends", Computer-Aided Design, Vol. 42, No. 12, pp. 1085-1094. doi: 10.1016/j.cad.2010.07.003.

Youcef-Toumi, K., Liu, W.S. and Asada H., 1987, "A Computer Integration of Reconfigurable Fixtures and Drilling of Sheet Metal Parts", Robotics and Factories of the Future '87, Proceedings of the 2nd International Conference San Diego, California, pp. 751-759. doi: 10.1007/978-3642-73890-6_90.

Yu, C., Li, D. and Li, X., 2011, "Application of the Preprocessing System of Stretch Forming Over Multi-Point Tooling in Real Factory Environment", Advanced Science Letters, Vol. 4, No. 6-7, pp. 2396-2399. doi: 10.1166/ asl.2011.1457.

Zhou, C., Cai, Z. and Li, M., 2005, "Stretching Process Based on Multi-Point Die and Its Numerical Simulation", Journal of Jilin University of Technology (Natural Science Edition), Vol. 35, pp. 287-291.

Zhou, F., 2007, "A New Generation Multi-Point Flexible Tooling for Aircraft Skin Stretch Forming", Aeronautical Manufacturing Technology, Vol. 11, pp. 30-33.

Zhou, K., Qian, Q. and Men, Y., 20०८, "A Robotized Reconfigurable Fixture", China patents 101269466. 